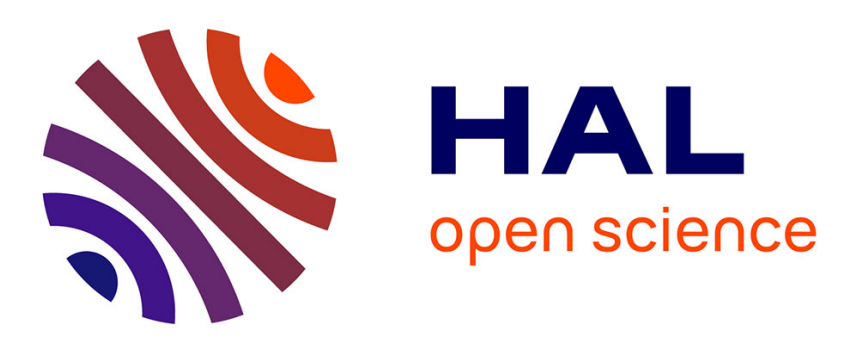

\title{
Reproductibilité du système métabolique Fitmate TM Cosmed pour mesurer la dépense énergétique sous maximale du pédalage
}

\author{
J Louis, Christophe Hausswirth, Jeanick Brisswalter
}

\section{To cite this version:}

J Louis, Christophe Hausswirth, Jeanick Brisswalter. Reproductibilité du système métabolique Fitmate TM Cosmed pour mesurer la dépense énergétique sous maximale du pédalage. Science \& Sports, 2012, 27 (3), pp.20-23. 10.1016/j.scispo.2011.10.012 . hal-01825925

HAL Id: hal-01825925

https://hal-insep.archives-ouvertes.fr/hal-01825925

Submitted on 28 Jun 2018

HAL is a multi-disciplinary open access archive for the deposit and dissemination of scientific research documents, whether they are published or not. The documents may come from teaching and research institutions in France or abroad, or from public or private research centers.
L'archive ouverte pluridisciplinaire HAL, est destinée au dépôt et à la diffusion de documents scientifiques de niveau recherche, publiés ou non, émanant des établissements d'enseignement et de recherche français ou étrangers, des laboratoires publics ou privés. 


\title{
Reproductibilité du système métabolique Fitmate ${ }^{\mathrm{TM}}$ Cosmed pour mesurer la dépense énergétique sous maximale du pédalage
}

\author{
J. Louis a, C. Hausswirth ${ }^{\text {b }}$, J. Brisswalter ${ }^{\text {a, }}$ \\ ${ }^{a}$ Laboratoire motricité humaine éducation sport santé (Lahmess), université de Nice Sophia-Antipolis, \\ 261, route de Grenoble, 06205 Nice cedex 3, France \\ ${ }^{\mathrm{b}}$ Service de recherche, Institut national du sport, de l'expertise et de la performance, 11, avenue du \\ Tremblay, 75012 Paris, France \\ * Auteur correspondant : Adresse e-mail : brisswalter@unice.fr (J. Brisswalter).
}

Article publié dans : Science \& Sports (ISSN 0765-1597), 2012, vol. 27, $\mathrm{n}^{\circ}$ 3, e20-e23 (doi:10.1016/j.scispo.2011.10.012)

\begin{abstract}
Résumé
Introduction. - Le but de cette étude est de vérifier la reproductibilité des mesures respiratoires au moyen du système métabolique Fitmate ${ }^{\mathrm{TM}}$, au cours d'un exercice de cyclisme sous maximal.

Synthèse des faits. - Vingt-et-une personnes ont effectué, lors de deux sessions, un exercice composé de trois fois cinq minutes de pédalage à des intensités sous maximales $(16,18$ et $21 \mathrm{~km} / \mathrm{h})$. La reproductibilité des mesures respiratoires était analysée entre les deux sessions.

Résultats. - Les coefficients de corrélation intraclasse (ICC) entre les deux sessions et pour les trois intensités varient de 0,61 à 0,71 pour $\mathrm{VO}_{2}$ et de 0,79 à 0,89 variant pour VE. Les coefficients de variation $(\mathrm{CV})$ sont inférieurs à $10 \%$ pour tous les paramètres et à toutes les vitesses. Ces résultats indiquent une bonne reproductibilité pour $\mathrm{VE}$ et une reproductibilité satisfaisante pour $\mathrm{VO}_{2}$ ou le rendement du pédalage.

Conclusion. -Le système Fitmate ${ }^{\mathrm{TM}}$ apparaît comme un système reproductible pour la mesure de VE et $\mathrm{VO}_{2}$ à des intensités sous maximales et permet ainsi l'évaluation de la dépense énergétique de façon rapide et aisée dans un cadre d'évaluation clinique.
\end{abstract}

Mots clés : Reproductibilité ; Consommation d'oxygène ; Cyclisme ; Analyseur métabolique portable

\begin{abstract}
Summary
Introduction. - The purpose of this study was to test the reproducibility of the Fitmate ${ }^{\mathrm{TM}}$ metabolic system in measuring breathing gas during a cycling exercise.

Synthesis of the facts. - Twenty-one participants performed during two sessions three 5-min exercises at submaximal intensities $(16,18$ et $21 \mathrm{~km} / \mathrm{h})$. The reproducibility of respiratory variables was assessed between the two sessions.

Results. - Intraclass correlation coefficient (ICC) between the two sessions and for all intensities ranged from 0.61 to 0.71 for $\mathrm{VO}_{2}$ and from 0.79 to 0.89 for VE. Coefficients of variation $\mathrm{CV}$ are always lower than $10 \%$ for all parameters and all speeds. These results indicate a good reproducibility for VE and a satisfactory reproducibility for $\mathrm{VO}_{2}$ and cycling efficiency.

Conclusion. - The Fitmate ${ }^{\mathrm{TM}}$ portable metabolic analyzer provides reproducible measures of $\mathrm{VO}_{2}$ and VE during steady-state exercise that allows the assessment of energy expenditure in a simple manner in a clinical framework.
\end{abstract}

Keywords: Reproducibility; Oxygen uptake; Cycling; Portable metabolic analyzer

\begin{abstract}
1. Introduction
La consommation en oxygène $\left(\mathrm{VO}_{2}\right)$ au repos ou à l'exercice est une des principales variables physiologiques étudiées pour identifier la charge métabolique du mouvement aussi bien dans une perspective d'évaluation clinique que dans un but d'optimisation de la performance sportive [1]. Sa mesure s'effectue grâce à des systèmes d'enregistrement fixes ou portables des gaz respirés. Ces systèmes sont valides et précis, mais souvent peu abordables à cause de leur coût élevé ou de leur utilisation compliquée. Le récent système métabolique Fitmate ${ }^{\mathrm{TM}}$ (Cosmed, Rome, Italie) représente
\end{abstract}


une alternative aux autres systèmes existants car il est de petite taille $(20 \times 24 \mathrm{~cm})$, peu cher et de fonctionnement accessible à tout praticien.

La précision du système métabolique Fitmate ${ }^{\mathrm{TM}}$ a déjà été validée scientifiquement aussi bien en conditions de repos que d'exercice [2]. Dans ces études, les valeurs de $\mathrm{VO}_{2}$ au repos ou au cours d'un exercice de course à pied d'intensité croissante sur tapis roulant étaient enregistrées avec le système métabolique Fitmate ${ }^{\mathrm{TM}}$ et comparées avec la méthode référence des sacs de Douglas. Aucune différence significative de valeurs de $\mathrm{VO}_{2}$ entre les deux méthodes de mesure aussi bien chez les hommes que chez les femmes n'a été relevée. Cependant, dans la perspective d'utiliser la mesure de $\mathrm{VO}_{2}$ afin d'identifier ou de valider des stratégies de réhabilitation clinique ou d'entraînement, il est nécessaire d'établir la reproductibilité des mesures. À ce jour, le critère de reproductibilité de la mesure de la reproductibilité du Fitmate ${ }^{\mathrm{TM}}$ n'a pas été établi.

Dans ce cadre, l'objectif de cette étude était de tester la reproductibilité du système métabolique Fitmate $^{\mathrm{TM}}$ au cours d'un exercice de cyclisme réalisé en laboratoire.

\section{Protocole expérimental}

\subsection{Sujets}

Vingt-et-une personnes ont accepté de participer à cette étude (âge : $34,6 \pm 12,4$ ans ; masse corporelle $: 66,8 \pm 11,4 \mathrm{~kg}$ ). Les participants étaient tous en bonne santé, physiquement actifs et pratiquaient régulièrement des activités d'endurance (durée d'entraînement : 4,6 $\pm 3,5 \mathrm{~h} /$ semaine). Ils ont été entièrement informés du protocole de l'étude et ont donné leur consentement écrit avant toute expérimentation. Le protocole a reçu l'accord du comité d'éthique local pour la protection des personnes.

\subsection{Exercice de cyclisme}

Tous les tests étaient réalisés sur un vélo (VAE, b’Twin 5.1, Oxylane, France), doté de 21 vitesses. La pression des pneus était contrôlée à quatre bars sur les conseils du fabriquant et pour s'assurer que la résistance liée aux frottements soit la même pour tous les sujets. Le protocole de cyclisme était entièrement réalisé sur un home-trainer (Elite s.r.l., Fontaniva, Italie) avec une résistance standardisée pour tous les sujets. Le braquet sélectionné sur le vélo était le même pour tous les sujets et pour toutes les conditions de pédalage, afin de ne pas influencer les variables enregistrées. La selle et le guidon étaient repositionnés selon les caractéristiques anthropométriques de chaque participant. Le vélo utilisé pour l'étude était aussi équipé d'un pédalier de type SRM (Schoberer Rad Messtechnik, Allemagne), muni de jauges de contraintes permettant d'enregistrer en continu la puissance de sortie (W), la cadence $(\mathrm{rpm})$ et la vitesse de pédalage $(\mathrm{km} / \mathrm{h})$.

Trois vitesses de pédalage ont été testées pendant une période de cinq minutes pour chaque vitesse et cinq minutes de récupération entre les vitesses. Trois vitesses $(16,18$ et $21 \mathrm{~km} / \mathrm{h})$ ont été imposées aux participants. Les vitesses étaient proposées dans un ordre aléatoire entre les sujets. Une seconde session identique était réalisée 24 heures après, à la même heure de la journée.

\subsection{Système de mesure des gaz respirés}

Le système métabolique FitmateTM est un appareil récent et compact $(20 \times 24 \mathrm{~cm})$, conc, u pour mesurer la consommation en oxygène, la dépense énergétique et la ventilation en conditions de repos et d'exercice. Il utilise une turbine munie d'un débitmètre pour mesurer la ventilation et une cellule combustible galvanique pour analyser la fraction en oxygène de l'air expiré. Le FitmateTM a un système de calibration automatique utilisant l'air ambiant et ne nécessite pas de seringue ou de gaz étalons. Cela est rendu possible par le fait que le FitmateTM ne dispose pas d'analyseur de $\mathrm{CO}_{2}$ et que le capteur d' $\mathrm{O}_{2}$ est intrinsèquement linéaire. Une seule calibration permet d'obtenir des valeurs pour l'ensemble de la séquence d'enregistrement. $\mathrm{VO}_{2}$ est calculée en utilisant l'équation suivante [2] :

$$
\begin{aligned}
\mathrm{VO}_{2}= & \left(\mathrm{FIO}_{2} \times \mathrm{IV}-\mathrm{FEO}_{2} \times \mathrm{EV}\right) \times \mathrm{FR} \times \mathrm{STPD} \\
& \cdot \text { où FR correspond à la fréquence respiratoire } ; \\
& \cdot \mathrm{IV}, \text { volume inspiré } ; \\
& \cdot \mathrm{EV}, \text { volume expiré } ; \\
& \cdot \mathrm{FIO}_{2}, \text { fraction inspirée en } \mathrm{O}_{2} ; \\
& \cdot \mathrm{FEO}_{2} \text {, fraction expirée en } \mathrm{O}_{2} \text {, dans des conditions standards (standard temperature } \\
& \text { and pressure }[\mathrm{STPD}])[2] .
\end{aligned}
$$




\subsection{Variables métaboliques mesurées}

$\mathrm{Au}$ cours de chaque séquence de cyclisme de cinq minutes, la consommation en oxygène $\left(\mathrm{VO}_{2}\right)$, le débit ventilatoire (VE), la fraction expirée en $\mathrm{O}_{2}\left(\mathrm{FEO}_{2}\right)$ et le quotient respiratoire $(\mathrm{QR})$ étaient enregistrés en continu et moyennés au cours des deux dernières minutes d'enregistrement pour obtenir un état stable.

Le rendement de la locomotion en cyclisme était aussi calculé en utilisant les valeurs de puissance développée et de dépense énergétique, selon la formule décrite par Gaesser et Brooks [3].

\subsection{Analyse statistique}

Les résultats sont exprimés selon la moyenne ()$\pm$ l'écart type $(\mathrm{ET})$. Une analyse de variance à deux voies (vitesse de pédalage $\times$ période) pour mesure répétée était d'abord réalisée pour évaluer l'effet éventuel du test et retest sur les variables dépendantes suivantes : $\mathrm{VO}_{2}, \mathrm{VE}, \mathrm{QR}$, gross efficiency (GR [i.e. rendement global]) cadence et la Puissance. L'ICC (ICC : rapport entre la variance intraclasse et la variance totale) était ensuite calculé. La reproductibilité est bonne quand l'ICC est compris entre 0,80 et 1,00, satisfaisant quand l'ICC est compris entre 0,60 et 0,79 , et faible quand l'ICC est inférieur à 0,60 . Enfin, le coefficient de variation $[\mathrm{CV}=(\mathrm{ET} /) \times 100]$ était calculé pour déterminer l'amplitude de variation des variables mesurées.

\section{Résultats}

Le Tableau 1 présente les valeurs des variables dépendantes, valeurs d'ICC et CV pour les trois vitesses considérées. Un effet significatif de la vitesse est observé sur les valeurs de cadence (F:2, 60 : $511,7, p<0,05)$ de $\mathrm{VO}_{2}(\mathrm{~F}: 2,60: 39,6, p<0,05)$ de $\mathrm{VE}(\mathrm{F}: 2,60: 21,1, p<0,05)$ de $\mathrm{QR}(\mathrm{F}: 2,60:$ $2,96, p<0,05)$ et de GE (F : 2, $60: 3,51, p<0,05)$. Aucune différence entre les deux tests n'est observée quelles que soient les vitesses. Les valeurs d'ICC montrent une bonne reproductibilité pour les valeurs de Puissance (ICC min-max : 0,90-0,96 ; CV min-max : 0,8-2,5\%), de cadence (ICC minmax : 0,96-0,97; CV min-max : 0,2-0,4 \%), de VE (ICC min-max : 0,79-0,89; CV min-max : 2,3-2,8 $\%$ ) et de QR (ICC min-max : 0,94-0,96; CV min-max : 0,2-0,6). La reproductibilité est satisfaisante pour les valeurs de $\mathrm{VO}_{2}$ (ICC min-max : 0,61-0,71; CV min-max : 4,3-6,8 \%) et de GE (ICC min-max : 0,60-0,64; CV min-max : 4,9-5,4\%).

\section{Discussion}

Le résultat principal de cette étude indique que le système Fitmate ${ }^{\mathrm{TM}}$ apparaît être reproductible de façon acceptable pour la mesure des paramètres gazeux et respiratoires à des intensités sous maximales. Très peu d'études ont été réalisées pour mesurer la reproductibilité des chaînes métaboliques de petites tailles alors que ces outils sembleraient être plus sensibles aux erreurs que les systèmes classiques fixes utilisés en laboratoire. [4]. Dans notre travail, nous relevons de valeurs d'ICC pour $\mathrm{VO}_{2}$ variant en fonction des vitesses de 0,61 à 0,71 et variant pour $\mathrm{VE}$ de 0,79 à 0,89 . Ces valeurs sont inférieures à celles relevées dans l'étude récente de Schrac et al. [5], qui ont comparé la reproductibilité d'un analyseur portable de type Cosmed K4b2 (Cosmed, Rome, Italie) et celle d'un analyseur fixe de type Medgraphics D-Series (St. Paul, États-Unis) à des intensités sous maximales. Dans cette étude les auteurs ont rapporté des valeurs d'ICC pour $\mathrm{VO}_{2}$ variant de 0,93 à 0,97 sans différence significative entre les deux appareils. Néanmoins, nos valeurs sont similaires à celles rapportées par Blessinger et al. [4], qui ont calculé des valeurs d'ICC de $\mathrm{VO}_{2}$ et de VE respectivement de 0,77 et 0,92 pour l'analyseur portable de type VmaxST (VIASYS, Yorba Linda, CA). Par ailleurs, les valeurs de CV reportées dans notre étude sont toutes inférieures à la valeur de $10 \%$, reconnue dans les études précédentes comme un critère minimal de reproductibilité acceptable $[4,6]$ et sont similaires à celles relevées avec un système fixe en laboratoire pour ce niveau d'intensité [1].

\section{Conclusion}

Le système Fitmate ${ }^{\mathrm{TM}}$ apparaît comme un système reproductible pour la mesure de $\mathrm{VE}$ et $\mathrm{VO}_{2}$ à des intensités sous maximales et permet ainsi l'évaluation de la dépense énergétique de façon rapide et aisée pour des intensités modérées. Cependant, l'absence de sonde à $\mathrm{CO}_{2}$ nécessite plus de prudence pour des intensités d'exercice intenses ou élevées.

\section{Déclaration d'intérêts}

Les auteurs déclarent ne pas avoir de conflits d'intérêts en relation avec cet article. 


\section{Références}

[1] Hausswirth C, Brisswalter J. Le coût énergétique de la course à pied de durée prolongée : étude des paramètres d'influence. Sci Sport 1999;14:59-70.

[2] Nieman DC, Lasasso H, Austin MD, Pearce S, McInnis T, Unick J. Validation of Cosmed's FitmateTM in measuring exercise metabolism. Res Sports Med 2007;15:67-75.

[3] Gaesser GA, Brooks GA. Muscular efficiency during steadyrate exercise: effects of speed and work rate. J Appl Physiol 1975;38:1132-9.

[4] Blessinger J, Sawyer B, Davis C, Irving BA, Weltman A, Gaesser G. Reliability of the VmaxST portable metabolic measurement system. Int J Sports Med 2009;30:22-6.

[5] Schrack JA, Simonsick EM, Ferrucci L. Comparison of the Cosmed K4b2 portable metabolic system in measuring steady-state walking energy expenditure. PLoS One 2010;5:e9292.

[6] Crouter SE, Antczak A, Hudak JR, Delta Valle DM, Haas JD. Accuracy and reliability of the ParvoMedics TrueOne 2400 and Medgraphics VO2000 metabolic systems. Eur J Appl Physiol 2006;98:139-51.

Tableau 1 Valeurs des paramètres mesurés lors des deux sessions et des paramètres de reproductibilité.

\begin{tabular}{|l|c|c|c|c|c|}
\hline & Intensités (km/h) & Session 1 & Session 2 & ICC & CV (\%) \\
\hline Puissance & & & & 0,90 & 1,19 \\
(watts) & 16 & $85,6 \pm 0,9$ & $85,5 \pm 0,8$ & 0,96 & 2,55 \\
& 18 & $101,8 \pm 0,95$ & $99,7 \pm 0,97$ & 0,95 & 0,89 \\
\hline Cadence (watts) & 21 & $120,9 \pm 0,9$ & $119,1 \pm 0,92$ & 0,96 & 0,2 \\
& 16 & $64,1 \pm 0,4$ & $54,4 \pm 0,2$ & 0,97 & 0,4 \\
& 21 & $70,1 \pm 0,3$ & $60,7 \pm 0,3$ & 0,97 & 0,3 \\
\hline VO2 (mL/min) & 16 & $1396,7 \pm 33$ & $1388,1 \pm 36$ & 0,66 & 4,3 \\
& 18 & $1531,7 \pm 34$ & $1546,8 \pm 37$ & 0,71 & 6,8 \\
\hline VE (L/min) & 21 & $1772,5 \pm 34$ & $1817,3 \pm 36$ & 0,61 & 2,7 \\
& 16 & $34,6 \pm 1,3$ & $34,5 \pm 1,3$ & 0,81 & 2,8 \\
\hline GE (\%) & 18 & $36,1 \pm 1,3$ & $37,8 \pm 1,2$ & 0,79 & 4,3 \\
& 21 & $45,1 \pm 1,1$ & $46,5 \pm 1,4$ & 0,89 & 5,1 \\
\hline QR & 16 & $18,1 \pm 0,3$ & $18,2 \pm 0,4$ & 0,60 & 5,4 \\
\hline & 18 & $19,5 \pm 0,3$ & $19,2 \pm 0,4$ & 0,64 & 0,6 \\
& $19,6 \pm 0,2$ & $19,4 \pm 0,3$ & 0,64 & 0,2 \\
\hline
\end{tabular}

ICC : coefficients de corrélation intraclasse ; CV : coefficient de variation ; QR : quotient respiratoire ; VE : débit ventilatoire; GE : gross efficiency (i.e. rendement global). 